\title{
NO ARBITRAGE UNDER TRANSACTION COSTS, WITH FRACTIONAL BROWNIAN MOTION AND BEYOND
}

\author{
PAOLO GUASONI \\ Boston University and University of Pisa
}

\begin{abstract}
We establish a simple no-arbitrage criterion which reduces the absence of arbitrage opportunities under proportional transaction costs to the condition that the asset price process may move arbitrarily little over arbitrarily large time intervals.

We show that this criterion is satisfied when the return process is either a Strong Markov process with regular points, or a continuous process with full support on the space of continuous functions. In particular, we prove that proportional transaction costs of any positive size eliminate arbitrage opportunities from Geometric Fractional Brownian Motion for $H \in(0,1)$ and with an arbitrary continuous deterministic drift.
\end{abstract}

KEY WORDS: Arbitrage, Transaction Costs, Fractional Brownian Motion, Markov Processes

\section{INTRODUCTION}

Absence of arbitrage is one of the most unifying concepts in Finance. Yet, empirical studies sometimes propose models for asset prices which are not consistent with this basic assumption. A case which has recently drawn much attention is Fractional Brownian Motion (fBm), which displays the long-range dependence observed in empirical data (Cutland, Kopp \& Willinger (1995), Willinger, Taqqu \& Teverovsky (1999) and the references therein). Since fBm is not a semimartingale (Liptser \& Shiryayev 1989, Maheswaran \& Sims 1993), the general results of Delbaen \& Schachermayer (1994) already imply that it allows some kind of free lunch. In fact, Rogers (1997), Salopek (1998), Shiryaev (1998), Dasgupta \& Kallianpur (2000) and Cheridito (2003) have shown that it allows outright arbitrage in a number of ways.

To bridge this chasm between theory and practice, one often cites market imperfections of various kinds, which may hinder arbitrageurs in their quest for riskless profits. For example, Salopek (1998) conjectured that that arbitrage with fBm would be eliminated by costly information or transaction costs. However, market imperfections usually lead to substantially more complicated models, and the effect on arbitrage possibilities is far from obvious. For instance, all explicit constructions of arbitrage for $\mathrm{fBm}$ involve trading very frequently, so one may expect that arbitrage disappears in a discrete-time, Donsker-type approximation of $\mathrm{fBm}$. On the contrary, arbitrage persists (Sottinen 2001). This result in turn suggests the presence of arbitrage for $\mathrm{fBm}$ when a minimal waiting time is required between subsequent transactions. But then arbitrage disappears (Cheridito 2003).

In this paper, which is originally motivated by the controversial case of Fractional Brownian Motion, we investigate the effect of transaction costs on arbitrage in more

Partially supported by the National Science Foundation under grant DMS-0532390 at Boston University.

I wish to thank Walter Schachermayer for useful remarks, Maurizio Pratelli for helpful discussions, and Laurent Decreusefond and Kostantinos Kardaras for pointing out errors in an earlier draft. I am especially indebted to the anonymous referee for a very perceptive reading, which led to substantial improvements. 
general models. We introduce a simple criterion, which shows that arbitrage opportunities disappear under transaction costs, as soon as the price process may remain within today's bid-ask spread over arbitrary (bounded) stochastic intervals. Motivated by this criterion, we define the related notion of stick) $]$ process, which implies the absence of arbitrage for arbitrarily small transaction costs and over arbitrarily large time horizons. Then we prove that stickiness is a very weak condition, which is satisfied by a large class of Markov processes encompassing most market models, including diffusions and Levy-processes. To handle $\mathrm{fBm}$, which is obviously not Markov, we show that a continuous process is sticky if it has full support. Then we exploit the characterization of the support of a Gaussian processes to verify that this condition is satisfied by $\mathrm{fBm}$.

The significance of these results is threefold: first, we show that absence of arbitrage with proportional transaction costs is achieved under minimal conditions. Indeed, we give several examples of asset price processes which allow for arbitrage in frictionless markets, but that become arbitrage-free under arbitrarily small transaction costs. Second, for Fractional Brownian Motion we obtain a framework which, acknowledging the presence of transaction costs, combines the sound economic interpretation of pathwise integrals (Björk \& Hult 2005) with the absence of arbitrage opportunities. Finally, we believe that these results will improve the understanding of absence of arbitrage under transaction costs, which is now clear in finite discrete time (see Kabanov, Rásonyi \& Stricker $(2001,2002,2003)$ and Schachermayer (2004)), but still lacks a definitive treatment in the general continuous-time case.

The rest of this paper is organized as follows: in the next section, we describe the market model in detail and introduce the main no-arbitrage criterion, which reduces absence of arbitrage to the stickiness of the asset price. In section 3 we prove this condition for a class of Markov processes with a simple argument based on excursions. An analogous result is proved in section 4 for the class of continuous processes with full support. In this case the proof is more technical, although the underlying idea is still quite simple. Section 5 deals with the specific case of (geometric) fBm with drift, which is shown to satisfy the previous condition. In the last section we consider possible extensions, and compare the present results to the (NA) and (NFLVR) conditions in (Delbaen \& Schachermayer 1994).

\section{NO ARBITRAGE WITH TRANSACTION COSTS}

We consider a market model with a riskless and a risky asset, based on a filtered probability space $\left(\Omega, \mathcal{F},\left(\mathcal{F}_{t}\right)_{t \in[0, \infty)}, P\right)$, where the filtration $\mathcal{F}_{t}$ satisfies the usual assumptions of right-continuity and saturatedness. The riskless asset is used as numeraire, and hence is assumed identically equal to 1 . The risky asset $X$ satisfies the following:

AsSUMPTION $2.1\left(X_{t}\right)_{t \in[0, \infty)}$ is a process with càdlàg (right-continuous with leftlimits) paths, strictly positive almost surely, adapted to $\mathcal{F}_{t}$, and quasi-left continuous with respect to the same filtration.

REMARK 2.2 The latter assumption of quasi-left continuity essentially requires that all jumps of $X$ are totally inaccessible, and is satisfied by all processes considered here, which are either continuous or satisfy the Strong Markov property, which implies quasileft continuity (Rogers \& Williams 2000, III.11).

An investor trades in the risky asset according to the strategy $\left(\theta_{t}\right)_{t \in[0, \infty)}$, which represents the number of shares held at time $t$, and each unit of numeraire traded in the

\footnotetext{
${ }^{1}$ Our terminology of sticky price is reminiscent of, but otherwise unrelated to, the usual dichotomy between flexible and sticky prices, which is obviously not new in macroeconomics.
} 
risky asset generates a transaction cost of $k$ units, which is charged to the riskless asset account. Consider a simple strategy $\theta$, which requires a finite number of transactions at stopping times $\left(\tau_{i}\right)_{i=1}^{n}$. Then $\theta=\sum_{i=1}^{n} \theta^{i} 1_{\left.] \tau_{i-1}, \tau_{i}\right]}$ for some random variables $\left(\theta^{i}\right)_{i=1}^{n}$, where $\theta^{i}$ is $\mathcal{F}_{\tau_{i}}$-measurable. We also conventionally set $\theta^{0}=0$. Then the liquidation value of a portfolio with zero initial capital is:

$$
V_{t}(\theta)=\sum_{i=1}^{n} \theta^{i-1}\left(X_{\tau_{i} \wedge t}-X_{\tau_{i-1} \wedge t}\right)-k \sum_{\tau_{i} \leq t} X_{\tau_{i}}\left|\theta^{i}-\theta^{i-1}\right|-k X_{t}\left|\theta_{t}\right|
$$

where the first term accounts for the capital gain, the second for the cost incurred in the various transactions, and the third for the final cost of liquidation. Passing from a finite number of transactions to continuous trading (that is, approximating $\theta$ with elementary strategies), under the quasi-left continuity assumption it is easy to see that the above quantity converges to (Guasoni 2002):

$$
V_{t}(\theta)=(\theta \cdot X)_{t}-k \int_{[0, t]} X_{s} d\|\theta\|_{s}-k X_{t}\left|\theta_{t}\right|
$$

where $\|\theta\|_{t}$ is the total variation of $\theta$ on $[0, t]$. Note that we are adopting the usual convention that $\theta_{0^{-}}=0$, so that the second term accounts also for the initial transaction. Finally, observe that the integral $(\theta \cdot X)_{t}$ above is defined pathwise, and coincides with the usual stochastic integral when $X$ is a semimartingale (Dellacherie \& Meyer 1982, VIII.1-2).

DEFINITION 2.3 An adapted, left-continuous (and hence predictable) process with paths of finite variation $\theta$ is an admissible strategy if $V_{t}(\theta) \geq-M$ a.s. for some $M>0$ and for all $t>0$. An admissible strategy is an arbitrage opportunity on $[0, T]$ if $V_{T}(\theta) \geq 0$ a.s. and $P\left(V_{T}(\theta)>0\right)>0$. A market is arbitrage free on $[0, T]$ if, for all admissible strategies $\theta, V_{T}(\theta) \geq 0$ a.s. only if $V_{T}(\theta)=0$ a.s.

REMARK 2.4 The above assumption that $\theta$ is adapted and left-continuous can be relaxed to $\theta$ predictable and of finite variation. However, when $X$ is quasi-left continuous, it turns out that $V(\theta)=V\left(\theta_{-}\right)$, therefore it is sufficient to consider adapted and left-continuous $\theta$ (see Guasoni (2002) for details).

We begin our investigation by observing that if some price process $\tilde{X}$ lies within the bid-ask spread of $X$, then trading on $X$ with transaction costs is not better that trading on $\tilde{X}$ without transaction costs. This is the message of the following Lemma, which is formulated pathwise, with no reference to probability:

LEMMA 2.5 Let $X, \tilde{X}:[0, \infty) \mapsto(0, \infty)$ be càdlàg functions such that:

$$
\left|X_{t}-\tilde{X}_{t}\right|<k X_{t} \text { for all } t \in[0, \infty)
$$

If $\theta:[0, \infty) \mapsto \mathbb{R}$ is a left-continuous function of bounded variation, then:

$$
V_{t}(\theta) \leq(\theta \cdot \tilde{X})_{t} \quad \text { for all } t \in[0, \infty)
$$

and equality holds for some $t$ if and only if $\theta_{s}=0$ for all $s \leq t$.

Proof. We have:

$$
V_{t}(\theta)=(\theta \cdot \tilde{X})_{t}+(\theta \cdot(X-\tilde{X}))_{t}-\int_{[0, t]} k X_{s} d\|\theta\|_{s}-k X_{t}\left|\theta_{t}\right|
$$

Recalling the convention $\theta_{0^{-}}=X_{0^{-}}=0$ and denoting by $\varepsilon_{t}=(X-\tilde{X})_{t}$, we obtain:

$$
(\theta \cdot(X-\tilde{X}))_{t}=(\theta \cdot \varepsilon)_{t}=\theta_{t} \varepsilon_{t}-\int_{[0, t]} \varepsilon_{s} d \theta_{s}
$$


where the "integration by parts" in the second equality follows by the definition of $(\theta \cdot \varepsilon)$ (Dellacherie \& Meyer 1982, VIII.1-2). From assumption (3) we have:

$$
\begin{aligned}
\theta_{t} \varepsilon_{t}-\left|\theta_{t}\right| k X_{t} & \leq 0 \\
-\int_{[0, t]} \varepsilon_{s} d \theta_{s}-\int_{[0, t]} k X_{s} d\|\theta\|_{s} & \leq 0
\end{aligned}
$$

which proves (4). To complete the proof, it suffices to observe that in (7) the left-hand side is strictly negative (by (3)) unless $\theta$ is constantly null on $[0, t]$.

REMARK 2.6 According to Lemma 2.5, the same strategy $\theta$ delivers a lower payoff when applied to $X$ with transaction costs than when applied to $\tilde{X}$ without transaction costs. Therefore the claims attainable with $X$ are dominated by those attainable with $\tilde{X}$, and any no-arbitrage condition which applies to $\tilde{X}$, will also extend to $X$. Hence we immediately obtain (somewhat artificial) examples of processes that are not semimartingales, but that are free of arbitrage under transaction costs. Consider a deterministic function $f$ with finite nonzero $p$-variation, with $p \in(1,2)$, and such that $\frac{1}{1+k}<f_{t}<\frac{1}{1-k}$ for all $t>0$ and some $k>0$. Then $X=f$ is obviously not a semimartingale, but satisfies Lemma 2.5 with $\tilde{X}=1$, and therefore it is certainly arbitrage-free.

REMARK 2.7 It is easy to see that Lemma 2.5 admits the following straightforward generalization: if $X, \tilde{X}$ are two processes such that:

$$
\left|X_{t}-\tilde{X}_{t}\right| \leq k_{t} X_{t}-\tilde{k}_{t} \tilde{X}_{t} \text { for all } t \in[0, \infty) \text { a.s. }
$$

then the payoff of $\theta$ on $X$ with transaction costs $k$ is lower than the payoff on $\tilde{X}$ with transaction costs $\tilde{k}$. The proof is analogous.

From Lemma 2.5 we obtain the general no-arbitrage criterion:

Proposition 2.8 Let $X$ satisfy Assumption 2.1] and let $k, T>0$. If, for all stopping times $\tau$ such that $P(\tau<T)>0$, we have that:

$$
P\left(\sup _{t \in[\tau, T]}\left|\frac{X_{\tau}}{X_{t}}-1\right|<k, \tau<T\right)>0
$$

then $X$ is arbitrage free with transaction costs $k$ in the interval $[0, T]$.

The main idea of the above criterion is the following: to achieve an arbitrage, at some point $(\tau)$ we have to start trading. This decision immediately generates a transaction cost which must be recovered at a later time, and this is possible only if the asset price moves enough in the future. Hence, if at all times there is a remote possibility of arbitrarily small price changes, then downside risk cannot be eliminated, and arbitrage is impossible. This idea can be made rigorous applying Lemma 2.5 to $\tilde{X}=X_{\tau \wedge t}$ :

Proof of Proposition 2.8. Consider a strategy $\theta$ which is not identically zero, and define the stopping time $\tau$ and the event $A$ as:

$$
\tau=T \wedge \inf \left\{t: \theta_{t} \neq 0\right\} \quad A=\left\{\sup _{t \in[\tau, T]}\left|\frac{X_{\tau}}{X_{t}}-1\right|<k, \tau<T\right\}
$$

By assumption, $P(\tau<T)>0$ and therefore $P(A)>0$ by $[8$. Using Lemma 2.5 with $\tilde{X}_{t} \equiv X_{t \wedge \tau}$, on the event $A$ we obtain that:

$$
V_{T}(\theta)=\int_{[\tau, T]} \theta_{s} d X_{s}-\int_{[\tau, T]} k X_{s} d\|\theta\|_{s}-k X_{T}\left|\theta_{T}\right|<0
$$


and hence $\theta$ is not an arbitrage.

The assumptions on $X$ in Proposition 2.8 seem weak enough to suggests that, in most cases, absence of arbitrage will hold for arbitrarily small transaction costs $k$ and over arbitrarily large horizons $T$. This is the case when the asset price is sticky:

DEFINITION 2.9 A progressively measurable process $Y$ is sticky with respect to the filtration $\mathcal{F}_{t}$ if, for all $\varepsilon, T>0$ and all stopping times $\tau$ such that $P(\tau<T)>0$, we have that:

$$
P\left(\sup _{t \in[\tau, T]}\left|Y_{\tau}-Y_{t}\right|<\varepsilon, \tau<T\right)>0
$$

COROLlary 2.10 Let $X$ satisfy Assumption 2.1. If $\log X$ is sticky, then $X$ is arbitrage free with transaction costs $k$ on the interval $[0, T]$, for all $k, T>0$.

Proof. If $X$ satisfies Assumption 2.1, it is progressively measurable since it is càdlàg and adapted, and also $Y_{t}=\log X_{t}$ is progressively measurable.

By the continuity in 0 of the function $x \mapsto\left|e^{x}-1\right|$, for all $k>0$ there exists some $\gamma>0$ such that, for all $T>0$ :

$$
\left\{\sup _{t \in[\tau, T]}\left|\frac{X_{\tau}}{X_{t}}-1\right|<k\right\}=\left\{\sup _{t \in[\tau, T]}\left|e^{Y_{\tau}-Y_{t}}-1\right|<k\right\} \supset\left\{\sup _{t \in[\tau, T]}\left|Y_{t}-Y_{\tau}\right|<\gamma\right\}
$$

and the claim follows.

\section{MARKOV PROCESSES}

In this section we show that a general class of Markov processes satisfies the stickiness condition (Definition 2.9. This is intuitively clear for the special case of Markov chains over a finite state space, where holding times are exponential, and hence take arbitrarily large values.

The point of view of excursions brings this idea to much greater generality. Holding times are replaced by waiting times for excursions larger than $\varepsilon$, which are still exponential, and essentially the same proof carries over.

The class of Strong Markov Processes considered in the next Proposition includes diffusion models, Levy processes, and Markov chains. In addition, it applies to singular diffusions such as $X_{t}=1+\left|B_{t}\right|$, where $B$ is a Brownian Motion (Revuz \& Yor 1994, III.1.14), (Karatzas \& Shreve 1991, 2.8.7), which allow for arbitrage opportunities in frictionless markets, but not under arbitrarily small proportional transaction costs.

Proposition 3.1 Let $X$ satisfy Assumption 2.1. and denote by $Y=\log X$. Suppose that there exists a family of probability laws $\left(P_{y}, y \in \mathbb{R}\right)$ such that:

i) $Y_{t}$ has the Strong Markov Property: for every finite $\mathcal{F}_{t}$-stopping time $\tau$, under the conditional law $P\left(\cdot \mid Y_{\tau}=y\right)$ the process $\left(Y_{\tau+t}\right)_{t \geq 0}$ is independent of $\mathcal{F}_{\tau}$ and has the law $P_{y}$.

ii) All points are regular: for all $y, P_{y}\left(\inf \left\{t>0: Y_{t}=y\right\}=0\right)=1$

Then $Y_{t}$ is sticky (Definition 2.9).

Note that the regularity condition $i$ i) above ensures the existence of local times at all points and hence the validity of the excursion argument given below. It also excludes trivial cases, such as deterministic Markov processes, which may allow arbitrage even with transaction costs. We refer to Rogers \& Williams (2000) for all definitions on Excursion Theory for Markov Processes. 
Proof of Proposition 3.1. By the Strong Markov property, we only need to prove the claim for $\tau=0, Y_{\tau}=y$, where $y$ is an arbitrary constant.

By the regularity assumption, at any point $y$ the process $Y$ admits a local time, that we denote by $L_{t}$. Also, we denote by $\tau_{l}=\inf \left\{t: L_{t}>l\right\}$ its right-continuous inverse, by $e_{l}$ the excursion process of $Y$ from $y$ at local time $l$, by $M(\xi)=\sup _{t>0}\left|\xi_{t}\right|$ the height of an excursion $\xi$, by $\Gamma_{\varepsilon}=\{\xi: M(\xi) \geq \varepsilon\}$ the set of excursions with height higher than $\varepsilon$ and by $N_{l}^{\varepsilon}=\sum_{s \leq l} 1_{\left\{e_{s} \in \Gamma_{\varepsilon}\right\}}$ their counting process.

By Itô's Theorem on excursions (Rogers \& Williams 2000, VI.47.6), $N_{l}^{\varepsilon}$ is a Poisson process. Moreover, its intensity $n\left(\Gamma_{\varepsilon}\right)$ has to be finite (Rogers \& Williams 2000, VI.48.1 (ii) with $G=(-\varepsilon, \varepsilon)$ ). It follows that:

$$
\begin{aligned}
P\left(\sup _{t \in[0, T]}\left|Y_{t}-y\right| \leq \varepsilon\right) \geq P & \left(\sup _{t \in\left[0, \tau_{L_{T}}\right]}\left|Y_{t}-y\right| \leq \varepsilon\right)=P\left(N_{L_{T}}^{\varepsilon}=0\right)= \\
& =E\left[E\left[1_{\left\{N_{L_{T}}^{\varepsilon}=0\right\}} \mid L_{T}\right]\right]=E\left[e^{-n\left(\Gamma_{\varepsilon}\right) L_{T}}\right]>0
\end{aligned}
$$

which completes the proof.

\section{CONTINUOUS PROCESSES WITH FULL SUPPORT}

The excursion argument given in the previous section applies to most models for asset price dynamics considered in the literature, but it obviously fails for $\mathrm{fBm}$, which is not a Markov process. To handle this case, which is the original motivation of the present paper, we now prove that stickiness (Definition 2.9) holds for a class of continuous processes characterized by the topological condition that the process is supported on the entire Wiener space. In particular, this condition is satisfied in the case of $\mathrm{fBm}$.

We denote the Wiener space by $\mathbb{W}=\{f \in C([0, \infty), \mathbb{R}): f(0)=0\}$, its trace on the interval $[0, t]$ by $\mathbb{W}_{t}=\{f \in C([0, t], \mathbb{R}): f(0)=0\}$, and the canonical projection $\mathbb{W} \mapsto \mathbb{W}_{t}$ by $\Pi_{t} . \mathbb{W}_{t}$ (resp. $\mathbb{W}$ ) is a Polish (metrizable complete separable) space with respect to the topology of uniform convergence (resp. uniform convergence on compact sets).

Recall that the support of a probability measure is the smallest closed set of probability one, and is always well-defined in the case of a Borel measure on a Polish space. In this section we prove the following:

PROPOSITION 4.1 Consider the canonical process $Y_{t}(\omega)=\omega_{t}$ on the space $(\mathbb{W}, \mathcal{B}(\mathbb{W}), P)$ filtered by the augmentation $\mathcal{F}_{t}$ of the natural filtration. If the support of $Y$ is $\mathbb{W}$, then $Y$ is sticky (Definition 2.9).

Before passing to the proof of Proposition 4.1, we briefly discuss its implications. Recall that a fundamental aspect of the notion of arbitrage is its invariance with respect to equivalent changes of probability. In other words, to acknowledge the presence of an arbitrage opportunity, two agents only need to agree on which events are possible or not, and not on their exact probabilities.

In the presence of transaction costs, and in the special but important case of a continuous process with its natural filtration, Proposition 4.1 implies an even greater robustness. In fact, agents only need to agree that all open events are possible, and absence of arbitrage will follow, regardless of disagreements on the possibility of more complex events.

When two processes are equivalent, they also have the same support, but the converse does not hold. For example, although Brownian Motion and Fractional Brownian 
Motion with $H \neq 1 / 2$ are mutually singular, in section 5 we show that they both are supported on the entire Wiener space.

A related consequence is the robustness of Proposition 4.1 with respect to changes of drift which do not necessarily preserve the equivalence of processes. In fact, if $f$ is a continuous function and $Y$ has full support, then also $Y+f$ does, regardless of the equivalence between $Y$ and $Y+f$. We illustrate this with the following:

EXAMPLE 4.2 Consider the process $Y_{t}=B_{t}+\sqrt{t}$, where $B$ is a Brownian Motion. It is clear that Brownian Motion is sticky (Definition 2.9), but this does not immediately imply the same property for $Y_{t}$, since $f: t \mapsto \sqrt{t}$ does not belong to the CameronMartin space:

$$
H=\left\{g: \int_{0}^{T}\left|g^{\prime}(s)\right|^{2} d s<\infty\right\}
$$

and therefore $Y$ and $B$ are mutually singular Gaussian Processes. Furthermore, in a frictionless market Delbaen \& Schachermayer (1995, Example 3.4) have shown that $Y_{t}=B_{t}+\sqrt{t}$ allows immediate arbitrage, and so does $X_{t}=e^{Y_{t}}$. However, since $Y$ is the translation of $B$ by a continuous function null at zero, it has the the same support as $B$, and Proposition 4.1 implies that for $X_{t}$ arbitrage disappears under transaction costs (in fact, a stronger statement holds, cf. Example 6.3 below).

We now turn to the proof of Proposition 4.1 The idea is to argue by contradiction: if a stochastic interval existed where small oscillations are forbidden, then some paths (precisely those which move little along this interval) would be excluded from the support of the process.

Before proceeding further, we need some more notation. Given an $\mathcal{F}$-measurable function $\xi$ and a $\sigma$-algebra $\mathcal{G} \subset \mathcal{F}$, we denote by $\operatorname{ess} \inf (\xi \mid \mathcal{G})$ the largest $\mathcal{G}$-measurable function smaller than $\xi$. Formally:

$$
\operatorname{ess} \inf (\xi \mid \mathcal{G})=\operatorname{ess} \sup \{g: g \text { is } \mathcal{G} \text {-measurable, } g \leq \xi \text { a.s. }\}
$$

For the definition and properties of ess sup, see for instance Karatzas \& Shreve (1998, Appendix A) or Föllmer \& Schied (2002, A.4). When $\mathcal{G}$ is trivial, the above definition coincides with the familiar essinf $\xi=\sup \{c: c \in \mathbb{R}, c \leq \xi$ a.s. $\}$. We need the following:

Lemma 4.3 Let $(U, \mathcal{U})$ and $(V, \mathcal{V})$ be Polish spaces such that $\mathcal{U}$ and $\mathcal{V}$ contain their respective Borel $\sigma$-algebras, and $P$ a probability on $U \times V$ with full support. Let $f$ : $U \times V \mapsto \mathbb{R}$ be continuous, uniformly in the second variable (i.e. the set $(f(\cdot, v))_{v \in \mathcal{V}}$ is equicontinuous). Then we have that:

$$
\operatorname{ess} \inf (f \mid \mathcal{U})=\inf _{v \in V} f(\cdot, v) \quad \text { a.s. in } P
$$

and the right-hand side is a continuous function $U \mapsto \mathbb{R}$.

REMARK 4.4 With a slight abuse of notation, in the left-hand side of 9 we have identified $\mathcal{U}$ with its image under the immersion of $U$ in $U \times V$.

Proof. Denote the left and right hand sides of (9) respectively by $\hat{f}$ and $\tilde{f}$, and also $P=P_{U} \otimes P_{V}$. We first show that $\tilde{f}$ is continuous. Since it is the infimum of a family of continuous functions, it is upper semicontinuous. To see that it is lower semicontinuous, consider a sequence $\left(u_{n}\right)_{n \geq 1} \subset U$ converging to $u$, and denote by $\left(v_{n}\right)_{n \geq 1} \subset V$ a sequence such that $f\left(u_{n}, v_{n}\right)<\tilde{f}\left(u_{n}\right)+\frac{1}{n}$. We have that:

$$
\tilde{f}(u) \leq f\left(u, v_{n}\right) \leq f\left(u_{n}, v_{n}\right)+\varepsilon_{n} \leq \tilde{f}\left(u_{n}\right)+\varepsilon_{n}+\frac{1}{n}
$$


where $\varepsilon_{n}$ converges to zero, and is independent of $v$ by uniform continuity. Passing to the limit, the claim follows.

Since $\tilde{f}$ is continuous, it is $\mathcal{U}$-measurable, and $\hat{f} \geq \tilde{f}$ almost surely. To see the reverse inequality, it suffices to find a sequence of $\mathcal{U}$-measurable functions $\phi_{n}: U \mapsto \mathbb{R}$ converging uniformly to $\tilde{f}$, and such that $\phi_{n} \geq \hat{f}$ almost surely. We define:

$$
f_{n}(u, v)=\min _{v \in V} 2^{-n}\left(\left\lfloor 2^{n} f(u, v)\right\rfloor+1\right)
$$

where $\lfloor x\rfloor$ denotes the integer part of $x$. Note that $f_{n}$ decreases uniformly to $f$, hence $\operatorname{ess} \inf \left(f_{n} \mid \mathcal{U}\right) \geq \operatorname{ess} \inf (f \mid \mathcal{U})$. Also, for all $(u, v)$ and $k \in \mathbb{Z}$ the set $A_{n}(k)=\{(x, y) \in$ $\left.U \times V: f_{n}(x, y)=k 2^{-n}\right\}$ contains an open set for infinitely many $n$. Hence we can define:

$$
f_{n}^{\prime}(u, v)=\min \left\{k 2^{-n}: k \geq f_{n}(u, v), A_{n}(k) \text { contains an open set }\right\}
$$

which still decreases uniformly to $f$ and has the additional property that all its level sets contain an open set. Since the support of $P$ is $U \times V$, all nonempty open sets have positive probability. Defining $\phi_{n}(u)=\min _{v \in V} f_{n}^{\prime}(u, v)$, we obtain that:

$$
\phi_{n}(u)=\operatorname{ess} \inf \left(f_{n}^{\prime} \mid \mathcal{U}\right) \geq \operatorname{essinf}(f \mid \mathcal{U})
$$

and since $\phi_{n}$ decreases to $\tilde{f}$, the thesis follows.

Proof of Proposition 4.1. With a slight abuse of notation, we identify $P$ with its image under the map $Y: \Omega \mapsto \mathbb{W}_{T}$, that is with a probability on the path space $\mathbb{W}_{T}$. We define the Lipschitz and hence uniformly continuous map $S: \mathbb{W}_{T} \mapsto \mathbb{W}_{T}$ :

$$
S_{t}(\omega)=\sup _{s \in[t, T]}\left|\omega_{s}-\omega_{t}\right|
$$

Then we need to prove that:

$$
P\left(S_{\tau}<\varepsilon, \tau<T\right)>0
$$

for all $\varepsilon>0$ and any stopping time $\tau$ such that $P(\tau<T)>0$. We now claim that:

$$
\operatorname{ess} \inf \left(S_{t} \mid \mathcal{F}_{t}\right)=0 \quad \text { a.s. for all } t \in[0, T]
$$

To see this, recall that $\Pi_{t}$ is the canonical projection on $\mathbb{W}_{t}$, and note that $\mathbb{W}_{T}$ can be identified with $\mathbb{W}_{t} \times \mathbb{W}_{T-t}$ through the application $I: \mathbb{W}_{T} \mapsto \mathbb{W}_{t} \times \mathbb{W}_{T-t}$ defined by $I(\omega)=\left(\Pi_{t}(\omega), \Pi_{T-t}\left(\omega \cdot-t-\omega_{t}\right)\right)$. Also, $\mathcal{F}_{t}$ coincides with the augmentation of the Borel $\sigma$-algebra of $\mathbb{W}_{t}$. Then we apply Lemma 4.3 with $U=\mathbb{W}_{t}, V=\mathbb{W}_{T-t}$ and $f(\omega, \eta)=S\left(I^{-1}(\omega, \eta)\right)_{t}$, and obtain almost surely:

$\operatorname{ess} \inf \left(S_{t} \mid \mathcal{F}_{t}\right)(\omega)=\inf \left\{S_{t}(\zeta): \zeta \in \mathbb{W}_{T}, \Pi_{t}(\zeta)=\Pi_{t}(\omega)\right\}=S_{t}\left(I^{-1}\left(\Pi_{t}(\omega), 0\right)\right)=0$ and therefore $P\left(S_{t}<\varepsilon \mid \mathcal{F}_{t}\right)>0$ a.s. for all $\varepsilon>0$ and $t \in[0, T]$. This fact immediately implies (10) for stopping times with at least an atom. In fact, if $P\left(t^{*}<T\right)>0$ for some $t^{*}$ we have that:

$$
P\left(S_{\tau}<\varepsilon, \tau<T\right) \geq P\left(S_{t^{*}}<\varepsilon, \tau=t^{*}\right)>0
$$

Now, suppose by contradiction that 10 is violated by some $\varepsilon>0$ and some stopping time $\tau$ such that $P(\tau<T)>0$, so that:

$$
\left\{S_{\tau} \geq \varepsilon, \tau<T\right\}=\{\tau<T\} \quad \text { a.s. }
$$

For fixed $n$, define $D=\left(T-2^{-k}\right)_{k \geq 1} \cup\left(k 2^{-n}\right)_{1 \leq k<2^{n} T}$, and the stopping time:

$$
\tau^{\prime}=\inf \left\{t>\tau: t \in D \text { or }\left|\omega_{t}-\omega_{\tau}\right|=\varepsilon / 2\right\}
$$


By continuity, for some large $n$ we have that $P\left(\tau^{\prime} \in D\right)>0$, therefore $\tau^{\prime}$ has atoms. Since by construction $\sup _{t \in\left[\tau, \tau^{\prime}\right]}\left|\omega_{t}-\omega_{\tau}\right| \leq \varepsilon / 2$, on the event $\{\tau<T\}$ we have:

$$
\varepsilon \leq S_{\tau}=\sup _{t \in\left[\tau^{\prime}, T\right]}\left|\omega_{t}-\omega_{\tau}\right| \leq \frac{\varepsilon}{2}+S_{\tau^{\prime}}
$$

and therefore $S_{\tau^{\prime}} \geq \varepsilon / 2$ on $\{\tau<T\}=\left\{\tau^{\prime}<T\right\}$. It follows that:

$$
0=P\left(S_{\tau^{\prime}}<\frac{\varepsilon}{2}, \tau^{\prime}<T\right)
$$

thus we reach a contradiction, since if $\tau^{\prime}$ has atoms and therefore (11) holds.

REMARK 4.5 At the beginning of this section we have observed that, if the support of $Y$ is the entire Wiener space, then the no-arbitrage property becomes robust with respect to arbitrary continuous deterministic changes of drift. For stochastic changes of drift, some care becomes necessary. For example, let $B$ be a Brownian Motion, and define $f_{t}=-\inf _{s \leq t} B_{s}$, which is a continuous increasing function. By Skorohod's Lemma, we have that $Y_{t}=B_{t}+f_{t}=\left|B_{t}\right|$, which is supported on positive functions only, while the support of $B_{t}$ is the entire Wiener space. However, one may obtain results involving stochastic drifts using the generalization of the Girsanov Theorem due to Bouleau \& Hirsch (1991), which allows for singular changes of measure. For the case of fBm, see also the paper of Norros, Valkeila \& Virtamo (1999).

\section{FRACTIONAL BROWNIAN MOTION}

Finally, we apply the criterion established in the previous section to prove that Geometric $\mathrm{fBm}$ is free of arbitrage with arbitrarily small transaction costs, and on arbitrarily large finite intervals. As a bonus of Proposition 4.1. we will obtain a certain freedom for the drift term. Recall that Fractional Brownian Motion with Hurst parameter $H \in(0,1)$ is a centered Gaussian process $\left(B_{t}^{H}\right)_{t \in[0, \infty)}$ with covariance $\Gamma_{H}(s, t)=\frac{1}{2}\left(s^{2 H}+t^{2 H}-|t-s|^{2 H}\right)$. We have the following:

Proposition 5.1 Let $Y_{t}=f_{t}+\sigma B_{t}^{H}$, where $\sigma>0$ and $f: \mathbb{R}_{+} \mapsto \mathbb{R}$ is a continuous function. Then $Y$ is sticky and thus for all $k, T>0, X_{t}=X_{0} e^{f_{t}+\sigma B_{t}^{H}}$ is arbitrage free with transaction costs $k$ on the finite interval $[0, T]$.

To prove this proposition, we shall use Theorem 3 in Kallianpur (1971), which states that the support of a Gaussian Process over a compact space and with continuous covariance function $\Gamma(s, t)$ is equal to the closure under the uniform norm of the corresponding Reproducing Kernel Hilbert Space (RKHS).

The RKHS of a centered Gaussian process indexed in $[0, T]$ with covariance $\Gamma(s, t)$ is the Hilbert space spanned by the functions of the form $\Gamma(\cdot, t)$ with the scalar product defined by $\langle\Gamma(\cdot, s), \Gamma(\cdot, t)\rangle=\Gamma(s, t)$. It is well-known (see Grenander (1981) for details), that if the covariance function can be written as:

$$
\Gamma(s, t)=\int_{0}^{T} K(s, u) K(t, u) d u
$$

for some square-integrable kernel $K$, then the RKHS is represented by:

$$
\mathbb{H}=\left\{\int_{0}^{\infty} K(t, u) g(u) d u: g \in L^{2}\left(\mathbb{R}_{+}\right)\right\}
$$

There are various characterizations of the RKHS of Fractional Brownian Motion in the literature, including Barton \& Poor (1988), Decreusefond \& Üstünel (1999) and Pipiras \& Taqqu (2000) (see Decreusefond (2003) for a recent survey). To handle the 
general case $H \in(0,1)$, we need a last piece of notation (cf. Remark 5.2 below). The Liouville fractional integral $I_{a^{+}}^{\alpha}$ is defined as (see Samko, Kilbas \& Marichev (1993)):

$$
\left(I_{a^{+}}^{\gamma} f\right)(t)=\frac{1}{\Gamma(\gamma)} \int_{a}^{t} f(s)(t-s)^{\gamma-1} d s
$$

Then Decreusefond \& Üstünel (1999, Corollary 3.1) have shown that (12) holds for Fractional Brownian Motion with a kernel $K_{H}$ which satisfies the equality:

$$
K_{H} f=\int_{0}^{\cdot} K_{H}(\cdot, s) f(s) d s=I_{0^{+}}^{2 H}\left(s^{1 / 2-H} I_{0^{+}}^{1 / 2-H}\left(s^{H-1 / 2} f\right)\right)
$$

The idea of the following proof, which is based on that of Theorem 3.3 in Decreusefond \& Üstünel (1999), is to combine the cited result of Kallianpur (1971) with (13), and conclude with the Stone-Weierstraß Theorem.

Proof of Proposition 5.1. We only need to show that she support of Fractional Brownian Motion on $[0, T]$ is $\mathbb{W}_{T}$, and the claim will follow from Proposition 4.1. For future reference, recall that for $a, b>0$ :

$$
\int_{0}^{t} s^{a-1}(t-s)^{b-1} d s=\frac{\Gamma(a) \Gamma(b)}{\Gamma(a+b)} t^{a+b-1}
$$

Let $f(s)=s^{\alpha}$. We have that:

$$
\begin{aligned}
I_{0^{+}}^{2 H}\left(s^{1 / 2-H} I_{0^{+}}^{1 / 2-H}\left(s^{H-1 / 2} f\right)\right) & =I_{0^{+}}^{2 H}\left(\frac{\Gamma\left(\alpha+H+\frac{1}{2}\right)}{\Gamma(\alpha+1)} s^{\alpha+1 / 2-H}\right)= \\
& =\frac{\Gamma\left(\alpha+H+\frac{1}{2}\right) \Gamma\left(\alpha+\frac{3}{2}-H\right)}{\Gamma(\alpha+1) \Gamma\left(\alpha+\frac{3}{2}+H\right)} s^{\alpha+H+\frac{1}{2}}
\end{aligned}
$$

Since we can freely choose $\alpha>-1$, by $[15)$ we have that the RKHS of fBm contains the algebra of all polynomials with order greater than one. This algebra separates $\mathbb{R}_{+} \backslash\{0\}$, and is null only at zero, and the Stone-Weierstraß Theorem implies that the RKHS of $\mathrm{fBm}$ is dense in $\mathbb{W}_{T}$. Thus Theorem 3 in Kallianpur (1971) implies that that the support of $B^{H}$ on $[0, T]$ is exactly $\mathbb{W}_{T}$.

REMARK 5.2 For $H \in(1 / 2,1)$, the above proof can be slightly simplified, using the RKHS characterization of Barton \& Poor (1988):

$$
\mathbb{H}=\left\{\int_{0}^{t} \int_{-\infty}^{s}(s-u)^{H-3 / 2} g(u) d u d s: g \in L^{2}(\mathbb{R})\right\}
$$

and then the same argument carries over. However, this result does not apply to the case $H \in(0,1 / 2)$, where fractional integrals seem inevitable.

REMARK 5.3 Corollary 2.10 hints at a connection between absence of arbitrage under transaction costs and the literature on small-ball probabilities (see Li \& Shao (2001) for a recent survey), which studies the rate of decay of:

$$
P\left(\sup _{u \in[s, t]}\left|Y_{u}-Y_{s}\right|<\varepsilon\right)
$$

as $\varepsilon$ decreases to zero. Precise results of this type are available for various classes of Gaussian processes $Y$ including fBm, therefore one wonders whether Proposition 5.1 can be proved quickly using these results instead. Indeed, for any stopping time $\tau \leq T$ we have that:

$$
\sup _{u \in[\tau, T]}\left|Y_{u}-Y_{\tau}\right| \leq\left|Y_{\tau}\right|+\sup _{u \in[\tau, T]}\left|Y_{u}\right| \leq 2 \sup _{u \in[0, T]}\left|Y_{u}\right|
$$


and therefore:

$$
A_{\varepsilon}=\left\{\sup _{u \in[\tau, T]}\left|Y_{u}-Y_{\tau}\right|<\varepsilon\right\} \supset\left\{\sup _{u \in[0, T]}\left|Y_{u}\right|<\frac{\varepsilon}{2}\right\}
$$

Thus, if the small-ball in the right-hand side has positive probability for all $\varepsilon>0$, then $P\left(A_{\varepsilon}\right)>0$ for all $\varepsilon>0$. However, in Corollary 2.9 we need a positive probability for the event $A_{\varepsilon} \cap\{\tau<T\}$, and not merely for $A_{\varepsilon}$, otherwise the argument in Proposition 2.8 fails. This is precisely the case when $\tau=T \wedge \inf \left\{t: \tilde{Z}_{t} \geq \varepsilon\right\}$ (see the proof of Proposition 4.1 for the definition of $\tilde{Z}$ ). Then we have that:

$$
0=P(A, \tau<T)<P(A)=P(\tau=T)
$$

Hence, in general, small-ball estimates do not guarantee the absence of arbitrage.

\section{6. (NA) VS. (NFLVR)}

In this paper we show that transaction costs often eliminate arbitrage opportunities otherwise present in frictionless models. The attentive reader probably noticed that our Definition 2.3 of arbitrage opportunity corresponds to the (NA) condition in frictionless markets, which is weaker than the (NFLVR) condition (Delbaen \& Schachermayer 1994). Then the question naturally arises if introducing transaction costs merely obtains the (NA) condition, or the stronger (NFLVR).

We begin with the following definition, given in analogy to the frictionless case:

Definition 6.1 Let $X$ satisfy Assumption 2.1] A Free Lunch with Vanishing Risk on $[0, T]$ is a sequence of admissible strategies $\left(\theta_{n}\right)_{n=1}^{\infty}$ such that:

i) $\lim _{n \rightarrow \infty} \operatorname{ess} \inf V_{T}\left(\theta_{n}\right)=0$

ii) $P\left(\lim _{n \rightarrow \infty} V_{T}\left(\theta_{n}\right)>0\right)>0$.

Thus $X$ satisfies (NFLVR) if, for any of converging sequence of admissible strategies $\left(\theta_{n}\right)_{n=1}^{\infty}$ satisfying $\left.i\right)$, we have that $\lim _{n \rightarrow \infty} V_{T}\left(\theta_{n}\right)=0$.

The following criterion is a straightforward consequence of Lemma 2.5 .

COROLlary 6.2 Let X satisfy Assumption 2.1. If there exists an adapted process $\tilde{X}$, such that:

i) $\tilde{X}$ satisfies (NFLVR) without transaction costs.

ii) $\sup _{t \in[0, T]}\left|1-\frac{\tilde{X}_{t}}{X_{t}}\right| \leq k$ almost surely.

Then $X$ satisfies (NFLVR) with transaction costs $k$ on $[0, T]$.

The problem with this criterion is that $\tilde{X}$ may not be easy to find, and this seems to be the case for Fractional Brownian Motion. On the other hand, this can be easily done in one of the examples previously considered:

EXAmple 6.3 Consider $Y_{t}=B_{t}+\sqrt{t}$, where $B$ is a Brownian Motion. In a frictionless market, this model does not satisfy (NA) as shown by Delbaen \& Schachermayer (1995, Example 3.4), and the same is true for $X_{t}=e^{Y_{t}}$. Since $Y$ is sticky, $X$ satisfies (NA) with arbitrarily small transaction costs. However, under the same assumptions we also have that (NFLVR) holds. Indeed, denote by $f_{t}^{\varepsilon}=e^{-\frac{\varepsilon}{t}} \sqrt{t}, Y_{t}^{\varepsilon}=B_{t}+f_{t}^{\varepsilon}$ and $X^{\varepsilon}=$ $e^{Y^{\varepsilon}}$. It is clear that $\sup _{t>0}\left|Y_{t}^{\varepsilon}-Y_{t}\right| \downarrow 0$ as $\varepsilon \downarrow 0$. Also, $Y^{\varepsilon}$ has a martingale measure, hence satisfies (NFLVR), and so does $X^{\varepsilon}$. Therefore the assumptions of Corollary 6.2 are satisfied, and $X$ satisfies (NFLVR) with arbitrarily small transaction costs. 


\section{REFERENCES}

Barton, R. J. \& Poor, H. V. (1988), 'Signal detection in fractional Gaussian noise', IEEE Trans. Inform. Theory 34(5, part 1), 943-959.

Björk, T. \& Hult, H. (2005), 'A note on wick products and the fractional black-scholes model', Finance Stoch. $9(2)$.

Bouleau, N. \& Hirsch, F. (1991), Dirichlet forms and analysis on Wiener space, Vol. 14 of de Gruyter Studies in Mathematics, Walter de Gruyter \& Co., Berlin.

Cheridito, P. (2003), 'Arbitrage in fractional Brownian motion models', Finance Stoch. 7(4), 533-553.

Cutland, N. J., Kopp, P. E. \& Willinger, W. (1995), Stock price returns and the Joseph effect: a fractional version of the Black-Scholes model, in 'Seminar on Stochastic Analysis, Random Fields and Applications (Ascona, 1993)', Vol. 36 of Progr. Probab., Birkhäuser, Basel, pp. 327-351.

Dasgupta, A. \& Kallianpur, G. (2000), 'Arbitrage opportunities for a class of Gladyshev processes', Appl. Math. Optim. 41(3), 377-385.

Decreusefond, L. (2003), Stochastic integration with respect to fractional Brownian motion, in 'Theory and applications of long-range dependence', Birkhäuser Boston, Boston, MA, pp. 203-226.

Decreusefond, L. \& Üstünel, A. S. (1999), 'Stochastic analysis of the fractional Brownian motion', Potential Anal. 10(2), 177-214.

Delbaen, F. \& Schachermayer, W. (1994), 'A general version of the fundamental theorem of asset pricing', Math. Ann. 300(3), 463-520.

Delbaen, F. \& Schachermayer, W. (1995), 'The existence of absolutely continuous local martingale measures', Ann. Appl. Probab. 5(4), 926-945.

Dellacherie, C. \& Meyer, P.-A. (1982), Probabilities and potential. B, Vol. 72 of North-Holland Mathematics Studies, North-Holland Publishing Co., Amsterdam. Theory of martingales, Translated from the French by J. P. Wilson.

Föllmer, H. \& Schied, A. (2002), Stochastic finance, Vol. 27 of de Gruyter Studies in Mathematics, Walter de Gruyter \& Co., Berlin. An introduction in discrete time.

Grenander, U. (1981), Abstract inference, John Wiley \& Sons Inc., New York. Wiley Series in Probability and Mathematical Statistics.

Guasoni, P. (2002), 'Optimal investment with transaction costs and without semimartingales', Ann. Appl. Probab. 12(4), 1227-1246.

Kabanov, Y. M. \& Stricker, C. (2001), 'The Harrison-Pliska arbitrage pricing theorem under transaction costs', J. Math. Econom. 35(2), 185-196. Arbitrage and control problems in finance.

Kabanov, Y., Rásonyi, M. \& Stricker, C. (2002), 'No-arbitrage criteria for financial markets with efficient friction', Finance Stoch. 6(3), 371-382.

Kabanov, Y., Rásonyi, M. \& Stricker, C. (2003), 'On the closedness of sums of convex cones in $L^{0}$ and the robust no-arbitrage property', Finance Stoch. 7(3), 403-411.

Kallianpur, G. (1971), 'Abstract Wiener processes and their reproducing kernel Hilbert spaces.', $Z$. Wahrscheinlichkeitstheorie und Verw. Gebiete 17, 113-123.

Karatzas, I. \& Shreve, S. E. (1991), Brownian motion and stochastic calculus, Vol. 113 of Graduate Texts in Mathematics, second edn, Springer-Verlag, New York.

Karatzas, I. \& Shreve, S. E. (1998), Methods of mathematical finance, Vol. 39 of Applications of Mathematics (New York), Springer-Verlag, New York.

Li, W. V. \& Shao, Q.-M. (2001), Gaussian processes: inequalities, small ball probabilities and applications, in 'Stochastic processes: theory and methods', Vol. 19 of Handbook of Statist., North-Holland, Amsterdam, pp. 533-597.

Liptser, R. S. \& Shiryayev, A. N. (1989), Theory of martingales, Vol. 49 of Mathematics and its Applications (Soviet Series), Kluwer Academic Publishers Group, Dordrecht. Translated from the Russian by K. Dzjaparidze [Kacha Dzhaparidze].

Maheswaran, S. \& Sims, C. A. (1993), Empirical implications of arbitrage-free asset markets, in P. Phillips, ed., 'Models, methods, and applications of econometrics: Essays in honor of A. R. Bergstrom', Basil Blackwell, pp. 301-316.

Norros, I., Valkeila, E. \& Virtamo, J. (1999), 'An elementary approach to a Girsanov formula and other analytical results on fractional Brownian motions', Bernoulli 5(4), 571-587.

Pipiras, V. \& Taqqu, M. S. (2000), 'Integration questions related to fractional Brownian motion', Probab. Theory Related Fields 118(2), 251-291.

Revuz, D. \& Yor, M. (1994), Continuous martingales and Brownian motion, Vol. 293 of Grundlehren der Mathematischen Wissenschaften [Fundamental Principles of Mathematical Sciences], second edn, Springer-Verlag, Berlin.

Rogers, L. C. G. (1997), 'Arbitrage with fractional Brownian motion', Math. Finance 7(1), 95-105. 
NO ARBITRAGE UNDER TRANSACTION COSTS, WITH FRACTIONAL BROWNIAN MOTION AND BEYOND3

Rogers, L. C. G. \& Williams, D. (2000), Diffusions, Markov processes, and martingales. Vol. 2, Cambridge Mathematical Library, Cambridge University Press, Cambridge. Itô calculus, Reprint of the second (1994) edition.

Salopek, D. M. (1998), 'Tolerance to arbitrage', Stochastic Process. Appl. 76(2), 217-230.

Samko, S. G., Kilbas, A. A. \& Marichev, O. I. (1993), Fractional integrals and derivatives, Gordon and Breach Science Publishers, Yverdon. Theory and applications, Edited and with a foreword by S. M. Nikol'skiř, Translated from the 1987 Russian original, Revised by the authors.

Schachermayer, W. (2004), 'The fundamental theorem of asset pricing under proportional transaction costs in finite discrete time', Math. Finance 14(1), 19-48.

Shiryaev, A. N. (1998), On arbitrage and replication for fractal models, Technical report, MaPhySto.

Sottinen, T. (2001), 'Fractional Brownian motion, random walks and binary market models', Finance Stoch. 5(3), 343-355.

Willinger, W., Taqqu, M. S. \& Teverovsky, V. (1999), 'Stock market prices and long-range dependence', Finance Stoch. 3(1), 1-13. 